\title{
International seminar on Drugs and Pregnancy held in Ebeltoft, Denmark, 1-2 May 1996
}

\section{Chairmen:}

Jørn Olsen (Aarhus, Denmark)

Lorentz Irgens (Bergen, Norway)

\section{Joint editor:}

Henrik Toft Sørensen (Aarhus, Denmark)

Seminar organisers: Henrik Toft Sørensen (Aarhus, Denmark) and Marianne Godt Hansen (Aarhus, Denmark)

\section{Participants:}

Jørn Olsen (Aarhus, Denmark); Henrik Toft Sørensen (Aarhus, Denmark); Gunnar Lauge Nielsen (Aalborg, Denmark); Weijin Zhou (Shanghai, China); Charlotte Søndergaard (Aarhus, Denmark); Charlotte Olesen (Aarhus, Denmark); Marianne Godt Hansen (Aarhus, Denmark); Lolkje de Jong-van den Berg (Groningen, Netherlands); Ingrid Matheson (Oslo, Norway); Patrick Leurquin (Brussels, Belgium); Liv Laegreid (Bergen, Norway); Andrew Czeizel (Budapest, Hungary); Thomas Bradley (Bromma, Sweden); Ulf Bergman (Bromma, Sweden); Rolv Terje Lie (Bergen, Norway); Lorentz Irgens (Bergen, Norway); Erik Lykke Mortensen (Copenhagen, Denmark)

The seminar was sponsored by the Danish Medical Research Council (grant no. $9600212 \mathrm{~kg} / \mathrm{mp}$ ) 